\title{
Migrated esophageal stent retrieved via oral double-balloon enteroscopy
}

Laith H. Jamil, MD, FASGE, FACG

A 72-year-old woman with a history of Billroth II surgery for peptic ulcer disease 18 years before presentation had experienced recurrent bezoar/gastric food retention secondary to a gastrojejunal (GJ) anastomotic stricture. Repeated dilation of the GJ anastomosis had been unsuccessful.

An 18-mm $\times 12$-cm esophageal through-the-scope, fully covered, self-expandable metallic stent (Taewoong Medical, Gimpo-si, Gyeonggi-do, South Korea) was placed across the surgical anastomosis and endoscopically sutured 3 times. A scheduled upper GI barium study 3 weeks later showed the stent to have migrated to the mid-distal jejunum (Fig. 1). The patient was asymptomatic; the stent was observed for 4 additional weeks by imaging studies and did not migrate further (Fig. 2).

Given the concern for potential adverse events, the patient was advised to have the stent retrieved (Video 1,

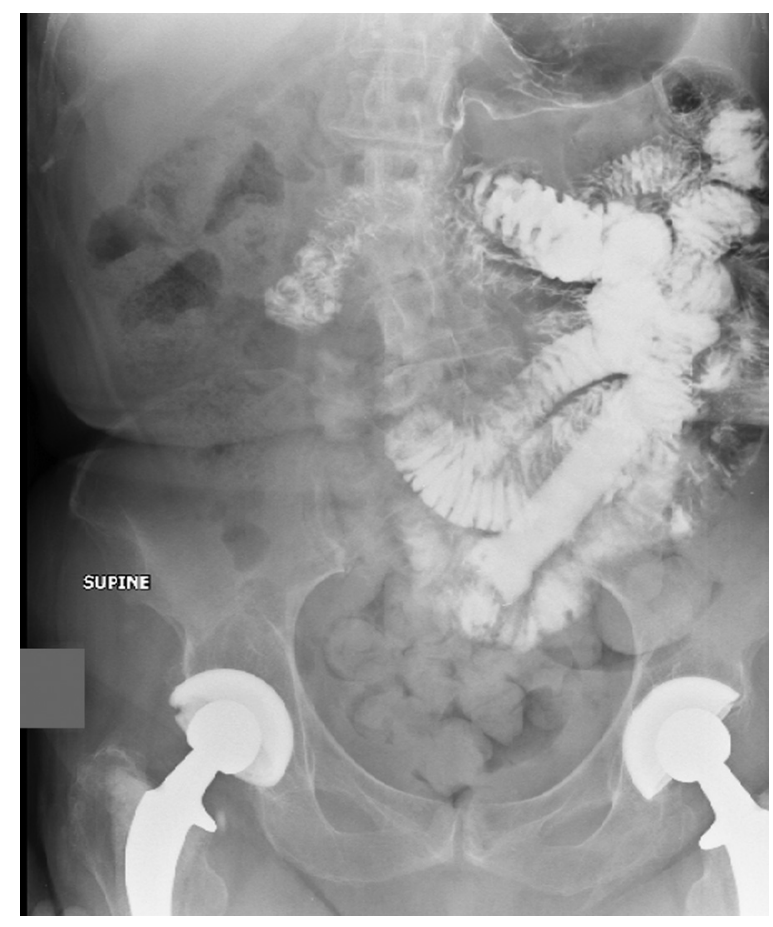

Figure 1. Upper GI barium study showing the esophgeal stent in the mid-distal jejunum. available online at www.VideoGIE.org). A Fujinon EN-450 T5 double-balloon enteroscope (Fujifilm, Tokyo, Japan) was advanced across a patent GJ anastomosis into the small bowel until the stent was reached, likely in the mid-distal jejunum. The internal thread on the upstream end of the stent was grasped with a rat-tooth/alligator grasping forceps (Endochoice DGC-383, Endochoice, Alpharetta, Ga, USA) and pulled inside the endoscope to collapse the proximal end of the stent (Fig. 3). Then, while the overtube was kept in place, the endoscope and stent were pulled through the overtube. The endoscope was then re-advanced through the overtube.

There were some superficial ulcerations, as well as a polypoid lesion with an ulcerated rim, likely reactive tissue from where the edge of the stent was embedded. Contrast medium injection visualized under fluoroscopy showed no evidence of extravasation (Fig. 4). Tattoo spot injection

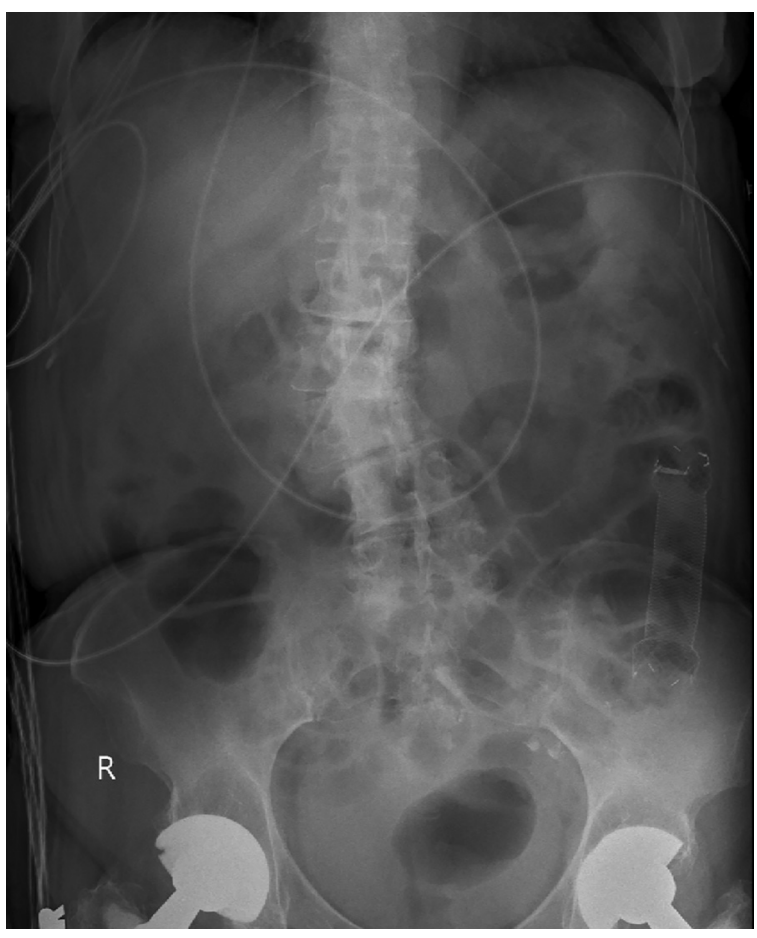

Figure 2. Abdominal radiograph showing the stent in the left lower quadrant.

Written transcript of the video audio is available online at www.VideoGIE.org. 


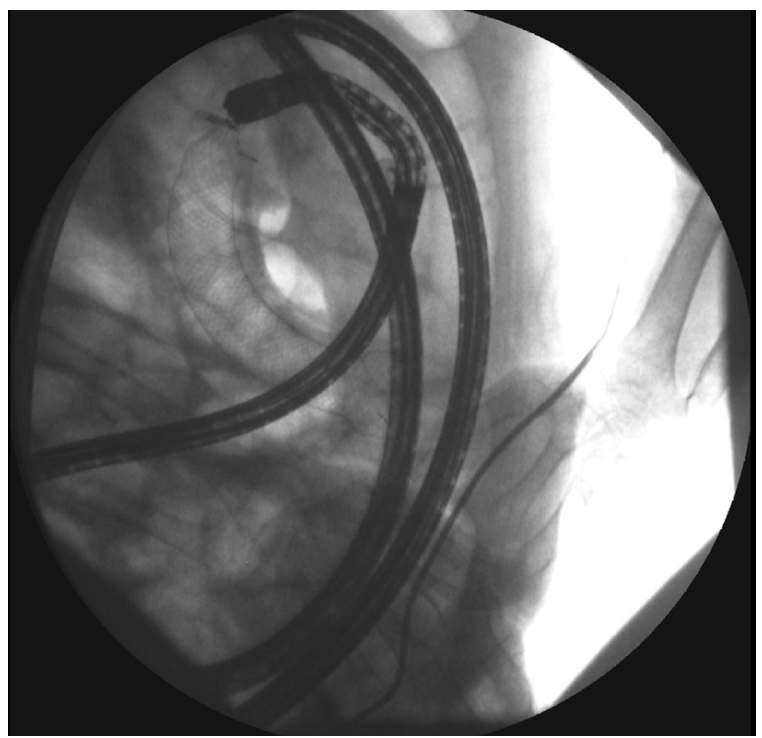

Figure 3. Fluoroscopy image showing the internal thread on the upstream end of the stent pulled inside the scope resulting in proximal collapse of the stent.

was performed approximately $2 \mathrm{~cm}$ upstream from the polypoid lesion as a reference point in the event a delayed adverse event was to occur.

The patient was discharged the following day with no symptoms. She underwent an ERCP 2.5 months later because of an episode of cholangitis. Her GJ anastomosis remained patent and was easily traversed. She was found to have a biliary stricture secondary to a well-differentiated adenocarcinoma arising within an intraductal papillary/tubular neoplasm of the common bile duct.

\section{DISCUSSION}

This case illustrates the use of the double-balloon enteroscope for removal of a migrated esophageal stent through the over-tube portion without causing harm to the small bowel. The endoscope can then be reintroduced to evaluate the area and ensure that there are

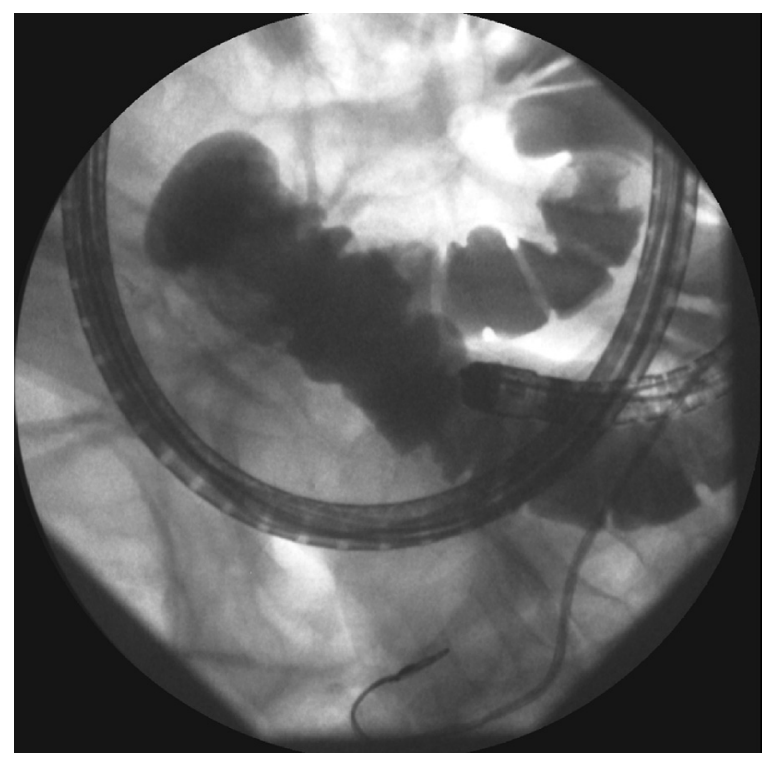

Figure 4. Contrast medium injection visualized under fluoroscopy showed no evidence of extravasation.

no adverse events or an underlying reason why the stent did not migrate spontaneously. Potentially, this technique can be used to remove other foreign bodies that migrate into the small bowel.

\section{DISCLOSURE}

The author disclosed no financial relationships relevant to this publication.

Abbreviation: GJ, gastrojejunal.

Cedars Sinai Medical Center, Los Angeles, California, USA.

Copyright $(2017$ The Authors. Published by Elsevier, Inc. on behalf of the American Society for Gastrointestinal Endoscopy. This is an open access article under the CC BY-NC-ND license (http://creativecommons.org/ licenses/by-nc-nd/4.0/).

http://dx.doi.org/10.1016/j.vgie.2016.12.010 